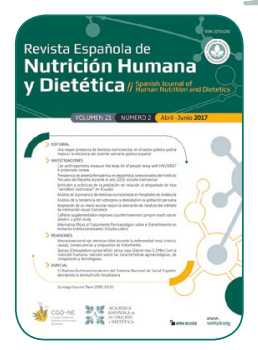

\title{
EDITORIAL
}

\section{Una mayor presencia de dietistas-nutricionistas en el sector público podría mejorar la eficiencia del sistema sanitario público español}

\author{
Alma Palau ${ }^{a, *}$, Giuseppe Russolillo \\ ${ }^{\mathbf{a}}$ Consejo General de Dietistas-Nutricionistas de España. \\ b Academia Española de Nutrición y Dietética. \\ *presidencia@consejodietistasnutricionistas.com
}

Recibido el 27 de junio de 2017; aceptado el 29 de junio de 2017; publicado el 30 de junio de 2017.

\section{CITA}

Palau A, Russolillo G. Una mayor presencia de dietistas-nutricionistas en el sector público podría mejorar la eficiencia del sistema sanitario público español. Rev Esp Nutr Hum Diet. 2017; 21(2): 98-100. doi: 10.14306/ renhyd.21.2.432

Esta es una de las conclusiones del primer borrador que se ha elaborado para celebrar el Día Mundial del Dietista-Nutricionista 2017 (24 de noviembre): "una mayor presencia de dietistas-nutricionistas en el Sistema Sanitario Español podría mejorar la eficiencia del sistema, por lo que se requiere un debate político urgente acerca de cómo aumentar su presencia en el sector público".

Según el informe que en 2013 estimó la carga de enfermedad global para 20151, los 5 factores de riesgo que más contribuyeron a la pérdida de años de vida debido a enfermedades, discapacidad o muerte temprana fueron por este orden: riesgos dietéticos, presión sistólica alta, malnutrición infantil y maternal, consumo de tabaco y polución del aire. Es decir, que el principal factor de riesgo de pérdida de años de vida es atribuible directamente a patrones de consumo de alimentos poco saludables.

Los factores de riesgo relacionados con la nutrición contribuyeron de forma significativa en el aumento de enfermedades de tres grupos: (a) enfermedades cardiovasculares y del sistema circulatorio; (b) cánceres y (c) diabetes, y enfermedades urogenitales, sanguíneas y endocrinas ${ }^{1}$.

Para revertir la tendencia de carga de enfermedad atribuible a factores dietéticos, deben realizarse acciones a todos los niveles: políticos (los gobiernos, legislación), económicos (la industria), y sociales (en educación y sensibilización, a nivel individual y profesional).

El papel de los/las dietistas-nutricionistas en el tratamiento y prevención de enfermedades crónicas y su inclusión en condiciones de equidad es un tema que requiere debate político urgente.

El Consejo General de Dietistas-Nutricionistas de España y la Academia Española de Nutrición y Dietética ostentan actualmente la representación de los/las dietistasnutricionistas de España en la International Confederation of Dietetic Associations - ICDA (nivel mundial), en la European 
Federation of the Associations of Dietitians - EFAD (nivel europeo), y en la Alianza Iberoamericana de Nutricionistas - AIBAN (nivel iberoamericano), y es conocedor a través de estas instituciones que la el/la dietista-nutricionista es una profesión sanitaria regulada, reconocida e incorporada en los sistemas nacionales de salud en la inmensa mayoría de países del mundo.

Actualmente, los/las dietistas-nutricionistas españoles/as están siendo formados en su mayoría en Universidades públicas, pero en cambio se ven obligados/as a ejercer principalmente en el sector privado o en otros países. Dicho de otra forma, por un lado con dinero público español se está formando a profesionales sanitarios que mejorarán la eficiencia del sistema sanitario de otros países. Por otro lado, a pesar de que los/las dietistas-nutricionistas que están trabajando en el sector privado están jugando un papel de primera línea y parcheando una clara deficiencia del sistema sanitario público español, dicha atención es sólo accesible para aquellos individuos que puedan pagarla, disminuyendo el potencial en resultados de salud en el sector de la sociedad que justamente más lo necesita -la población con bajos ingresos-, y poniendo en riesgo la equidad en el acceso a una mejor asistencia sanitaria. Es hora de que el gobierno tome cartas en el asunto y se proponga realizar un estudio para aumentar la presencia del dietista-nutricionista en sus equipos de trabajo.

El borrador ofrece otra conclusión que debe ser tomada en cuenta: "si bien existen muchos trabajos que evalúan la eficacia y el coste-eficacia de las intervenciones dietéticas, son mucho más escasos los trabajos que evalúan el impacto de la inclusión del dietista-nutricionista en equipos interdisciplinares, especialmente en cuanto al impacto económico".

A pesar de las limitaciones en las evaluaciones económicas del impacto delaumento dela presencia del dietista-nutricionista, el borrador recoge datos de revisiones sistemáticas que apuntan a una mejora de la eficiencia del sistema sanitario. Por ejemplo, una revisión sistemática publicada en $2015^{2}$, concluye que los/as dietistas que trabajan en atención primaria de salud pueden ser una apuesta coste-efectiva, potencialmente ahorrando al sistema de salud 5,8€-105€ por cada $1 €$ gastado en la intervención dietética (que incluye el salario de este profesional). En relación a entornos terapéuticos (hospitalario, principalmente), a pesar de que los datos del impacto económico de la inclusión del dietistanutricionista son aún más escasos, después de obtener pruebas que indican que las intervenciones dietéticas son coste-eficaces ${ }^{3-13}$, que la inclusión de dietistas-nutricionistas en equipos interdisciplinares (versus no inclusión) aumenta la eficacia de las intervenciones ${ }^{14-16}$, y tener en cuenta los datos ofrecidos por una modelización realizada por la Dutch Association of Dietitians ${ }^{17}$ y los datos de 5 estudios evaluados por la Evidence Analysis Library of the Academy of Nutrition and Dietetics ${ }^{18}$, parece probable concluir que la inclusión de dietistas-nutricionistas españoles en los equipos interdisciplinares del sistema nacional de salud podría mejorar la eficiencia del sistema (mayor eficacia con menor gasto sanitario).

Otra conclusión que ofrece el borrador es la necesidad de más investigación en relación a la evaluación de la presencia, eficacia y coste-eficacia de la figura del/la dietistanutricionista en todos los sectores en los que participa. En el presente número de la Revista Española de Dietistas, cabe destacar la presencia de 2 artículos relacionados con el tema.

En el estudio de revisión de Benítez ${ }^{19}$ se subraya que a pesar de que en España uno de cada cuatro pacientes hospitalizados está en riesgo de desnutrición o desnutrido, porcentaje que aumenta si el paciente es mayor, en muy pocos hospitales españoles se atienden adecuadamente las necesidades nutricionales de los pacientes o se cuenta con personal especializado y medios para valorar nutricionalmente a los pacientes y, además, la codificación de la desnutrición es prácticamente nula en los informes de alta. En este sentido, la incorporación de dietistas-nutricionistas al Sistema Nacional de Salud, especialmente en atención primaria y especializada, ofrecería la posibilidad de combatir la desnutrición hospitalaria en todos sus niveles, pero además ofrecería seguridad y eficacia en los servicios relacionados con la atención nutricional ${ }^{19}$. Esto lleva a entender que la pronta actuación mediante tratamiento nutricional podría reducir costes económicos por su efecto atenuante sobre ciertos parámetros (estancia media, reingreso y complicaciones) ${ }^{19}$.

En este sentido, ya en 2009, la Asociación Española de Dietistas-Nutricionistas (AEDN) hizo una propuesta al Senado español para la inclusión de dietistas-nutricionistas en el Servicio Nacional de Salud, donde se exponían las principales necesidades y propuestas de reforma para que se llevasen a cabo $^{20}$. A pesar de ello y del interés público que suscita este colectivo profesional, en 2017, la presencia de esta figura en hospitales públicos y privados en España sigue siendo deficiente, incumpliéndose los criterios de la Declaración de la AEDN. Para poder disponer de una visión más clara de la situación actual, se hace necesaria la presencia de estudios que describan la presencia de este colectivo en la red hospitalaria española y que evalúen en qué medida se cumplen los criterios de la citada Declaración de Postura de la AEDN. A día de hoy, sólo se dispone del dato de Andalucía, gracias al estudio de García-Puche y Col. ${ }^{21}$, cuya conclusión determina que la presencia de dietistasnutricionistas en hospitales públicos y privados de Andalucía incumple los criterios de la Declaración de Postura de la AEDN sobre la incorporación del colectivo en el Sistema Nacional de Salud. Por ello, hacen falta más estudios para observar la situación en las demás comunidades autónomas y describir la situación de esta figura profesional en este ámbito 21 . 
Animamos a que más estudios como los presentes en este número, sean enviados y evaluados para su publicación en la Revista Española de Nutrición Humana y Dietética, y recordamos que existen grandes vacíos en cuanto a la evaluación de la presencia, eficacia y coste-eficacia de la figura del/la dietista-nutricionista españoles en todos los sectores en los que participa.

\section{CONFLICTO DE INTERESES}

AP es presidenta del Consejo General de Dietistas-Nutricionistas de España y GR es presidente de la Academia Española de Nutrición y Dietética.

\section{REFERENCIAS}

(1) Forouzanfar MH, Afshin A, Alexander LT, Anderson HR, Bhutta ZA, Biryukov S, et al. Global, regional, and national comparative risk assessment of 79 behavioural, environmental and occupational, and metabolic risks or clusters of risks, 19902015: a systematic analysis for the Global Burden of Disease Study 2015. Lancet. 2016; 388(10053): 1659-724.

(2) Howatson A, Wall CR, Turner-Benny P. The contribution of dietitians to the primary health care workforce. J Prim Health Care. 2015; 7(4): 324-32.

(3) Tuffaha HW, Roberts S, Chaboyer W, Gordon LG, Scuffham PA. Cost-effectiveness Analysis of Nutritional Support for the Prevention of Pressure Ulcers in High-Risk Hospitalized Patients. Adv Skin Wound Care. 2016; 29(6): 261-7.

(4) Tuffaha HW, Roberts S, Chaboyer W, Gordon LG, Scuffham PA. Cost-effectiveness and value of information analysis of nutritional support for preventing pressure ulcers in high-risk patients: implement now, research later. Appl Health Econ Health Policy. 2015; 13(2): 167-79.

(5) Banks MD, Graves N, Bauer JD, Ash S. Cost effectiveness of nutrition support in the prevention of pressure ulcer in hospitals. Eur ] Clin Nutr. 2013; 67(1): 42-6.

(6) Doyle E, Simmance N, Wilding H, Porter J. Systematic review and meta-analyses of foodservice interventions and their effect on nutritional outcomes and satisfaction of adult oncology patients. Nutr Diet. 2017; 74(2): 116-28.

(7) Alouki K, Delisle H, Bermúdez-Tamayo C, Johri M. Lifestyle Interventions to Prevent Type 2 Diabetes: A Systematic Review of Economic Evaluation Studies. ] Diabetes Res. 2016; 2016: 2159890

(8) Elia M, Normand C, Laviano A, Norman K. A systematic review of the cost and cost effectiveness of using standard oral nutritional supplements in community and care home settings. Clin Nutr. 2016; 35(1): 125-37.

(9) Elia M, Normand C, Norman K, Laviano A. A systematic review of the cost and cost effectiveness of using standard oral nutritional supplements in the hospital setting. Clin Nutr. 2016; 35(2): 370-80.
(10) Tsertsvadze A, Gurung T, Court R, Clarke A, Sutcliffe P. Clinical effectiveness and cost-effectiveness of elemental nutrition for the maintenance of remission in Crohn's disease: a systematic review and meta-analysis. Health Technol Assess. 2015; 19(26): 1-138.

(11) Mitchell $H$, Porter J. The cost-effectiveness of identifying and treating malnutrition in hospitals: a systematic review. J Hum Nutr Diet. 2016; 29(2): 156-64.

(12) Freijer K, Bours MJL, Nuijten MJC, Poley MJ, Meijers JMM, Halfens RJG, et al. The economic value of enteral medical nutrition in the management of disease-related malnutrition: a systematic review. J Am Med Dir Assoc. 2014; 15(1): 17-29.

(13) Pradelli L, Povero M, Muscaritoli M, Eandi M. Updated cost-effectiveness analysis of supplemental glutamine for parenteral nutrition of intensive-care patients. Eur ] Clin Nutr. 2015; 69(5): 546-51.

(14) Karavetian M, de Vries N, Rizk R, Elzein H. Dietary educational interventions for management of hyperphosphatemia in hemodialysis patients: a systematic review and meta-analysis. Nutr Rev. 2014; 72(7): 471-82.

(15) Riegel GR, Ribeiro PAB, Rodrigues MP, Zuchinali P, Moreira LB. Efficacy of nutritional recommendations given by registered dietitians compared to other healthcare providers in reducing arterial blood pressure: Systematic review and meta-analysis. Clin Nutr. 2016.

(16) Munk T, Tolstrup U, Beck AM, Holst M, Rasmussen HH, Hovhannisyan $K$, et al. Individualised dietary counselling for nutritionally at-risk older patients following discharge from acute hospital to home: a systematic review and meta-analysis. J Hum Nutr Diet. 2016; 29(2): 196-208.

(17) Lammers M, Kok L. Cost-benefit analysis of dietary treatment [Internet]. Amsterdam, Países Bajos: Seo Economic Research; 2012 [consultado: 26/06/2017]. Disponible en: http://www. seo.nl/uploads/media/2012-76a_Cost-benefit_analysis_of_ dietary_treatment.pdf

(18) Academy of Nutrition and Dietetics. MNT: Cost Effectiveness, Cost-benefit, or Economic Savings of MNT (2009) [Internet]. Academy of Nutrition and Dietetics - Evidence Analysis Library. 2009 [consultado: 26/06/2017]. Disponible en: https://www. andeal.org/topic.cfm?menu=3949\&cat $=4085$

(19) Benítez N. El Dietista-Nutricionista dentro del Sistema Nacional de Salud Español: abordando la desnutrición hospitalaria. Rev Esp Nutr Hum Diet. 2017; 21(2): 199-208.

(20) Russolillo G, Baladia E, Moñino M, Colomer M, García M, Basulto J, et al. Incorporación del dietista-nutricionista en el Sistema Nacional de Salud (SNS): Declaración de Postura de la Asociación Española de Dietistas-Nutricionistas (AEDN). Act Diet. 2009; 13(2): 62-9.

(21) García-Puche A, Cabañas-Alite L. Análisis de la presencia de dietistas-nutricionistas en hospitales de Andalucía. Rev Esp Nutr Hum Diet. 2017; 21(2): 130-36. 